\title{
Deformation nanotwins suppress shear banding during impact test of CrCoNi medium-entropy alloy
}

\author{
Xiaolei Wu ${ }^{\mathrm{a}, \mathrm{b}, *}$, Muxin Yang ${ }^{\mathrm{a}}$, Ping Jiang ${ }^{\mathrm{a}}$, Chang Wang ${ }^{\mathrm{c}}$, Lingling Zhou ${ }^{\mathrm{a}}$, Fuping Yuan ${ }^{\mathrm{a}, \mathrm{b}}$, \\ Evan Ma ${ }^{\mathrm{d}}$ \\ a State Key Laboratory of Nonlinear Mechanics, Institute of Mechanics, Chinese Academy of Sciences, 15 Beisihuan West Road, Beijing 100190, China \\ ${ }^{\mathrm{b}}$ School of Engineering Science, University of Chinese Academy of Sciences, Beijing 100049, China \\ ${ }^{c}$ Central Laboratory, Central Iron and Steel Research Institute, 76 Xueyuan South Road, Beijing 100081, China \\ ${ }^{\mathrm{d}}$ Department of Materials Science and Engineering, Johns Hopkins University, Baltimore, MD 21218, United States
}

\section{A R T I C L E I N F O}

\section{Article history:}

Received 2 October 2019

Revised 13 December 2019

Accepted 13 December 2019

Available online 24 December 2019

\section{Keywords:}

Nano-twin

Shear-band

Strain hardening

Impact toughness

Medium-entropy alloy

\begin{abstract}
A B S T R A C T
We report deformation bands ahead of the crack tip in a CrCoNi alloy under impact loading, and analyze the effects of deformation twinning on strain localization in the form of shear banding. Due to low stacking fault energy, upon high-strain-rate deformation this medium-entropy alloy forms twins with thickness and spacing of only a few nanometers. These nano-twins are embedded dynamically not only ahead of any developing shear band to retard its advance, but also in its interior to work harden. This dual effect helps to suppress runaway shear banding instability that instigates failure and contributes to the energy absorption as recorded in instrumented Charpy tests.
\end{abstract}

(C) 2019 Acta Materialia Inc. Published by Elsevier Ltd. All rights reserved.
High mode I fracture toughness $\left(\mathrm{K}_{\mathrm{IC}}\right)$, rivaling those of the best stainless steels, has recently been reported for some face-centeredcubic (FCC) high-entropy alloys (HEAs) and medium-entropy alloys (MEAs) at room temperature (RT, $293 \mathrm{~K}$ ) and liquid-nitrogen temperature (LNT, $77 \mathrm{~K}$ ) [1,2]. In addition to $\mathrm{K}_{\mathrm{IC}}$, some HEAs/MEAs also show good impact fracture toughness $\left(A_{K}\right)$ in Charpy tests [3-5]. Such high toughness has been attributed to deformation twinning (DT): the incessant formation of nano-twins (NTs) in these alloys, together with a high density of dislocations inside twin lamellae, carries large plasticity and induces pronounced strain hardening [6-8], consuming energy and leading to toughening $[9,10]$. As these FCC HEAs/MEAs have relatively low yield strengths and high strain hardening capabilities, the fracture resistance mainly arises from the development of a large plastic deformation zone ahead of the crack tip.

While the above paints a global picture for the high toughness against crack initiation and propagation, the details surrounding the possible plastic instability and incipient damage have rarely been discussed. The first question is, even in such ductile HEAs/MEAs, does strain localization leading to shear bands (SBs) happen upon impact loading at high strain rates? Secondly,

\footnotetext{
* Corresponding author.

E-mail address: xlwu@imech.ac.cn (X. Wu).
}

if SBs get to emerge, do they instigate damage to initiate and sustain cracking $[5,11,12]$ ? If the answer is yes, then what is the exact mechanism that puts brakes on the shear banding plastic instability? Note that when SBs develop violently without control, there would be much less chance for spread-out deformation to consume energy throughout the plastic zone. Therefore, the plastic events associated with stabilized SBs are expected to be a contributor to the overall toughness. A question naturally arises as to how the DT (see last paragraph) helps to stabilize SBs (when they do get to form)? Detailed microstructural information down to nanometer scale in and around the localized deformation bands (all generally referred to as SBs hereafter) is therefore useful.

The microstructural response inside a SB is usually dominated by dislocation plasticity along with dynamic recrystallization, in most metals with moderate to high stacking fault energy (SFE) [13-15]. However, in a low SFE material (such as the CrCoNi MEA [16]) if profuse NTs can form dynamically in and around the SB, strain hardening can be superior to that due to dislocation activities [6]. So far there has been no analysis regarding this detail, one reason being that bulk samples with adequate dimensions are often not readily available to meet standard $A_{K}$ test requirements. In non-impact (such as quasi-static) tests, even though DT happens from time to time $[1,2,17,18]$, the thickness/spacing of the claimed NTs is actually not truly down to nanometer scale in most cases. 
a

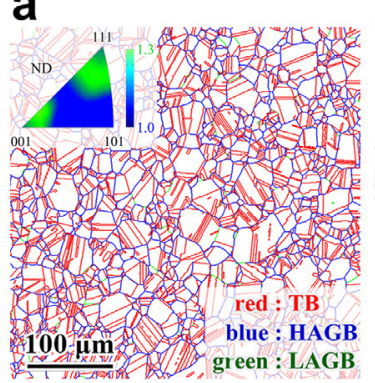

b

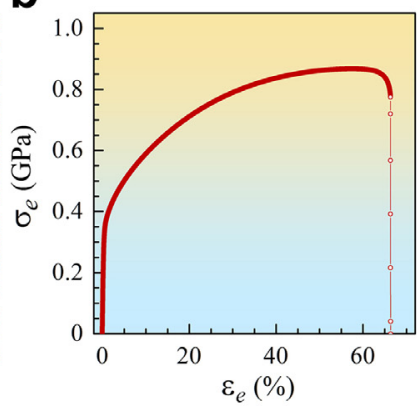

C

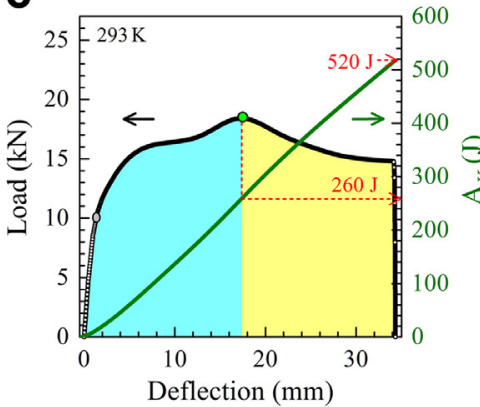

d

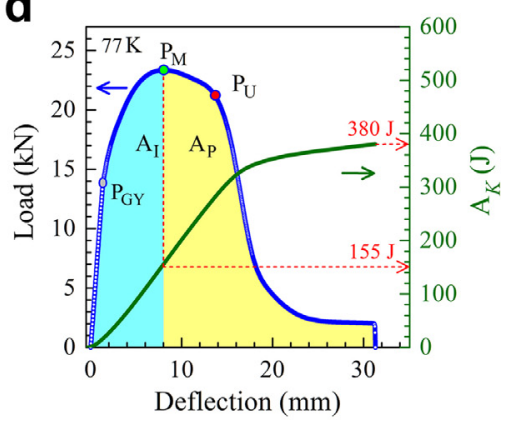

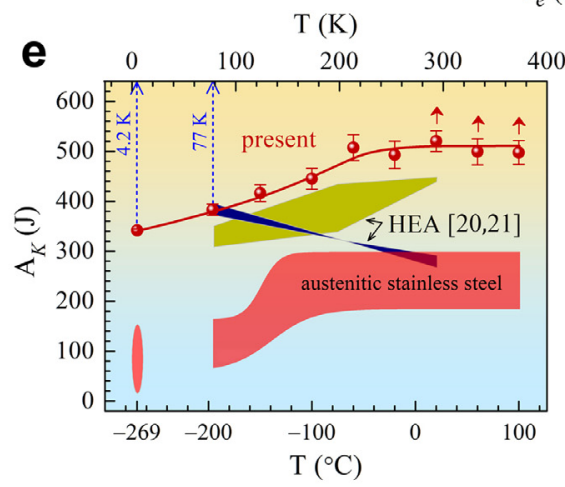

$\mathbf{f}$
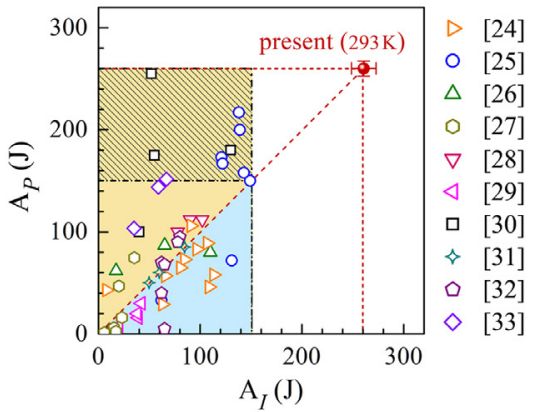

g

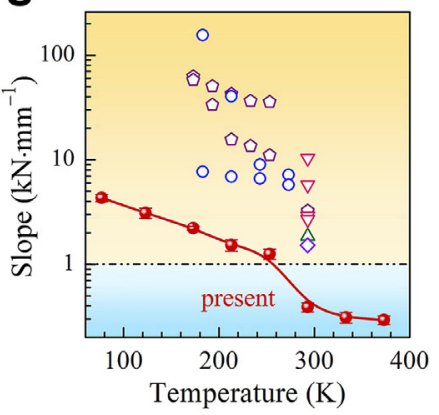

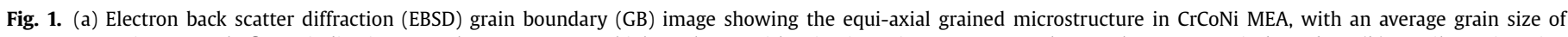

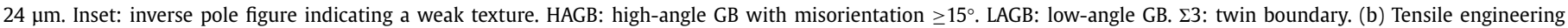

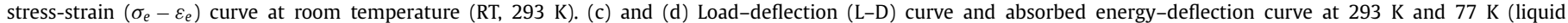

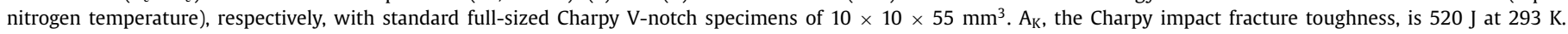

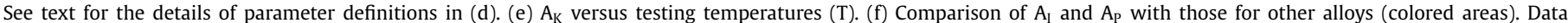

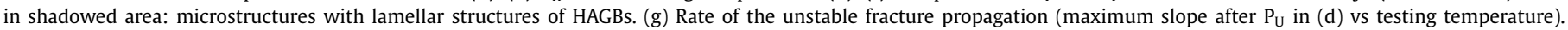
(For interpretation of the references to colour in this figure legend, the reader is referred to the web version of this article.)

In the following, we present a detailed account of the dual role of DT in bringing shear banding instability under control during Charpy impact tests of ternary CrCoNi MEA. DT accumulates NTs not only ahead of the tip of an advancing SB, but also inside the SB to work harden. Hence, any SB that gets induced by impact loading can self-toughen, via DT induced strain hardening both inside and outside the band. In addition, we report $A_{K}$ values measured not only for standard-sized samples, but also using an instrumented Charpy tester which provided quantitative load-deflection (L-D) curves and absorbed energy-deflection curves. The energy consumption recorded, together with microscopic images at and around the SB tip of the deformation nanotwins that are as fine as a few nanometers in their thickness and spacing, provide additional information and insight into the origin of the instability/damage tolerance.

The equi-atomic CrCoNi MEA was produced by the electromagnetic levitation melt technique [19]. The chemical composition was $30.7 \mathrm{Cr}-34.7 \mathrm{Co}-34.6 \mathrm{Ni}$ (wt.\%). The ingot was homogenized at $1473 \mathrm{~K}$ for $12 \mathrm{~h}$ and closed-die forged to obtain samples of $12 \mathrm{~mm}$ thick, followed by subsequent annealing at $1173 \mathrm{~K}$ for $2 \mathrm{~h}$. The standard full-size of Charpy V-notch (CVN) impact specimens was $10 \times 10 \times 55 \mathrm{~mm}^{3}$, with the $\mathrm{V}$-notch of radius of $0.25 \pm 0.025 \mathrm{~mm}$. The CVN specimens were tested using an NCS NI750C instrumented Charpy pendulum impact tester of $750 \mathrm{~J}$ capacity, equipped with an instrumented Charpy impulse data acquisition system according to ASTM E23 standard. Five specimens were used for $A_{K}$ tests and the presented data are the average with standard deviation. The microstructure characterization data presented in this paper are mostly for samples tested at RT $(293 \mathrm{~K})$, but $A_{K}$ values and curves also include those at other test temperatures. The quasi-static tensile tests, used to obtain a baseline stress-strain curve, were conducted at strain rate of
$5 \times 10^{-4} \mathrm{~s}^{-1}$ at RT. The microstructure was characterized using an electron backscatter diffraction (EBSD) imaging and transmission electron microscopy. The site-specific TEM foil samples (e.g. near the tip of either the shear-band or crack and near the fracture surface) were cut from the longitudinal-sectional of fractured CVN samples using focused ion beam (FIB) micro-machining.

Fig. $1 \mathrm{a}$ is the EBSD grain boundary (GB) image, showing that after recrystallization annealing, this $\mathrm{CrCoNi}$ MEA exhibits equi-axed grains with an average grain size of $26 \mu \mathrm{m}$. Fig. $1 \mathrm{~b}$ is the tensile engineering stress-strain $\left(\sigma_{e}-\varepsilon_{e}\right)$ curve at RT. Fig. 1c shows the load-deflection (L-D) curve and absorbed energy-deflection curve derived from the instrumented $A_{K}$ tester at RT. For comparison, the results are also given for tests at LNT (77 K), see Fig. $1 \mathrm{~d}$. The vertical line on the right-side of L-D curve indicates that the pendulum loses contact with CVN specimen during impact loading, bending and flying off the gap ( $40 \mathrm{~mm}$ wide) of the impact tester.

Fig. 1e shows the Charpy impact fracture toughness, $A_{K}$, in the temperature range between $373 \mathrm{~K}$ and $77 \mathrm{~K}$. The $A_{K}$ (total impact absorbed energy) is $520 \mathrm{~J}$ at $293 \mathrm{~K}$ (Fig. 1c) and $380 \mathrm{~J}$ at $77 \mathrm{~K}$ (Fig. 1d); these high values are comparable to the best HEAs reported recently $[5,20,21]$. Our instrumented Charpy measurements, however, allow the $A_{K}$ to be analyzed in terms of L-D curves with several characteristic loads and absorbed energies [22,23]. As illustrated in Fig. $1 \mathrm{~d}, \mathrm{P}_{\mathrm{GY}}$ and $\mathrm{P}_{\mathrm{M}}$ are, respectively, the general yield load and maximum load, and $\mathrm{P}_{\mathrm{U}}$ indicates the onset of unstable fracture propagation. $A_{K}$ consists of energy absorption in two stages. $A_{I}$, namely the area under the L-D curve up to $P_{M}$, represents the amount of energy absorbed before the initiation of a sharp crack at the notch. That is, $A_{I}$ indicates how difficult the crack initiation is. The remaining area, $A_{P}$, represents both the stable (area under $\mathrm{L}-\mathrm{D}$ curve between $\mathrm{P}_{\mathrm{M}}$ and $\mathrm{P}_{\mathrm{U}}$ ) and unstable (area 
a

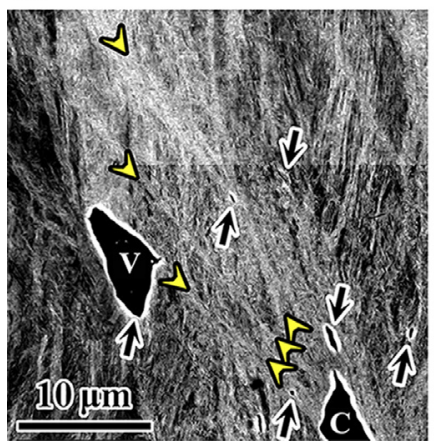

b

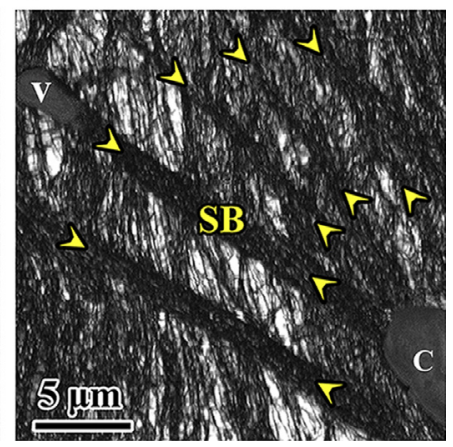

Fig. 2. (a) SEM image showing deformation features around the tip of the main crack (labeled by $C$ ) in a CVN sample after $A_{K}$ testing at RT. Shear-bands (SBs): white thin lines emitted from crack tip (labelled by swallow-tailed double-headed yellow arrows). Micro-voids: v labeled by arrows. (b) Close-up view showing the emission of SBs from the tip of the crack (labeled by C). Note the presence of a micro-void (labeled by $\mathrm{v}$ ) on the advancing path of the SB. (For interpretation of the references to colour in this figure legend, the reader is referred to the web version of this article.)

after $\mathrm{P}_{\mathrm{U}}$ ) fracture propagation energy. The slope after $\mathrm{P}_{\mathrm{U}}$ characterizes the rate of unstable crack propagation.

Note the presence of the maximum load point, $\mathrm{P}_{\mathrm{M}}$, in the loaddeflection (L-D) curve (see Fig. 1c and d) during instrumented impact testing. It has been normally regarded that $\mathrm{P}_{\mathrm{M}}$, i.e. the onset of load drop, is actually the demarcation point of crack initiation and crack propagation [24]. In other words, both the shear-banding and the ensuing stable crack propagation along the shear-bands should begin at $\mathrm{P}_{\mathrm{M}}$. Here, the initiated crack is exactly the reason of load drop. Under impact loading at high strain rate, the shear-banding is the precursor of cracking. In other words, shear bands have already formed inside the plastic zone at $\mathrm{P}_{\mathrm{M}}$, and they mediate the subsequent propagation of crack.

Several distinct features of impact responses are noted for this CrCoNi MEA. First, both $A_{I}$ and $A_{P}$ (see Fig. 1f) reach nearly twice of those (all data selected from standard full-size CVN samples for $A_{K}$ testing) in alloys of dislocation plasticity (colored area) [24-33]. Furthermore, $A_{P}$ is comparable to and even much higher than those of lamellar structures (shadow area). Second, $\mathrm{P}_{\mathrm{U}}$ is unavailable in RT test (Fig. 1c), in contrast to the clear signal at LNT (Fig. 1d). This indicates that the unstable crack propagation does not get to develop at RT due to strong suppression of crack propagation. Third, the slope after $\mathrm{P}_{\mathrm{U}}$ at RT (Fig. $1 \mathrm{~g}$ ) is also at least one order of magnitude smaller than those of other alloys characterized by dislocation plasticity.

Fig. 2a is a SEM image showing the morphology ahead of the tip of the main crack (labeled by C) in the fractured CVN sample tested at RT, showing several narrow SBs (white thin lines, labeled by swallow-tailed double-headed yellow arrows), along with micro-voids (arrows). These are plastic instability that can develop into precursors for a propagating crack in a ductile material [11,12]. In general, cracking tends to follow the path of the advancing SB, where strain localization incurs damage via the emergence, growth and coalescence of micro-voids. Fig. $2 \mathrm{~b}$ is a close-up view of SBs in the area ahead of the crack tip. A micro-void (labeled by $v$ ) is indeed visible inside the SB. These SBs emit from the crack tip where large stress concentration exists.

TEM observations show that DT serves as the dominant mode for both plastic deformation and work hardening upon impact loading at RT. Fig. 3a is a TEM image showing NTs of high density activated in two twin systems, at $0.1 \mathrm{~mm}$ away from the crack tip. These are primary NTs, typically running across the whole grain. Dislocations tangles of high density exist near and at the twin boundaries (TBs). Upon impact loading, both the complex stress state near the $\mathrm{V}$-notch and high strain rate $\left(\sim 10^{3} \mathrm{~s}^{-1}\right)$ facilitate a

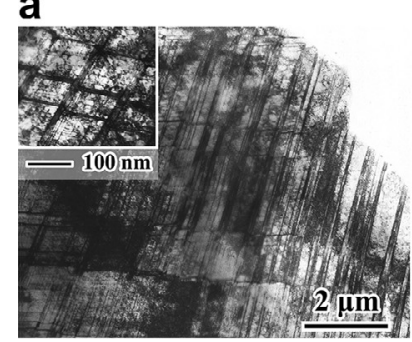

d

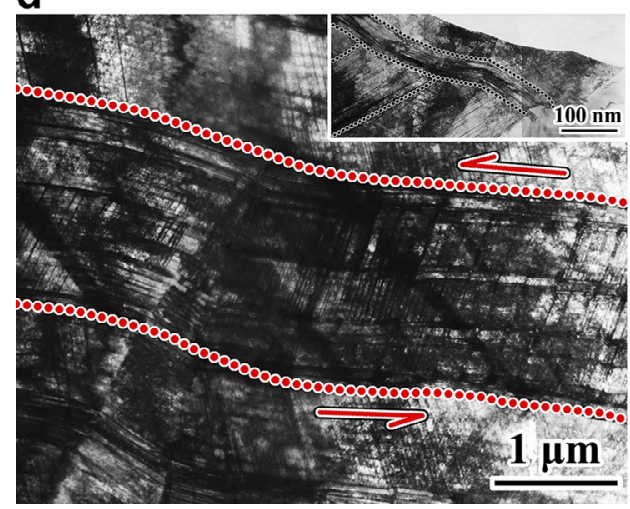

b

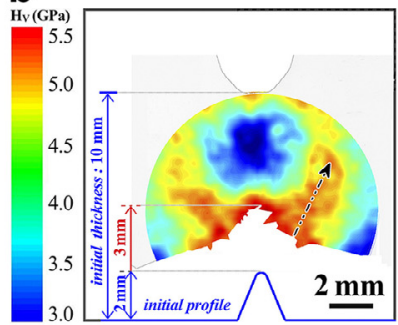

c

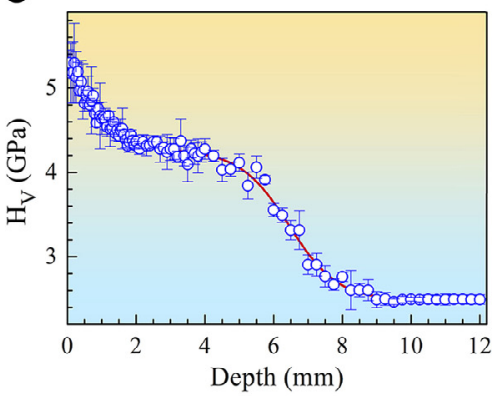

e

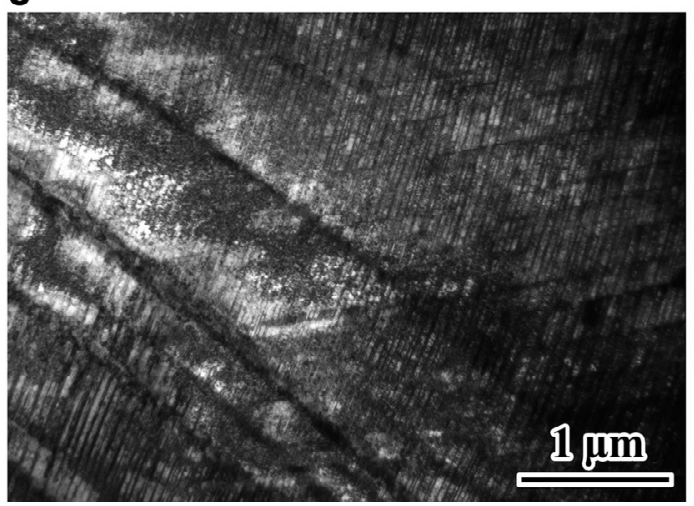

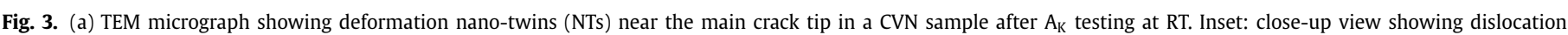

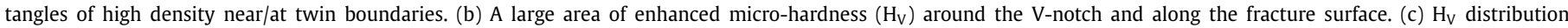

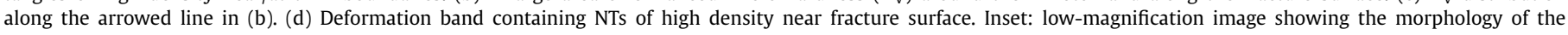
band. (e) Deformation NTs ahead of and at a distance of $\sim 1 \mathrm{~mm}$ away from the crack tip. 


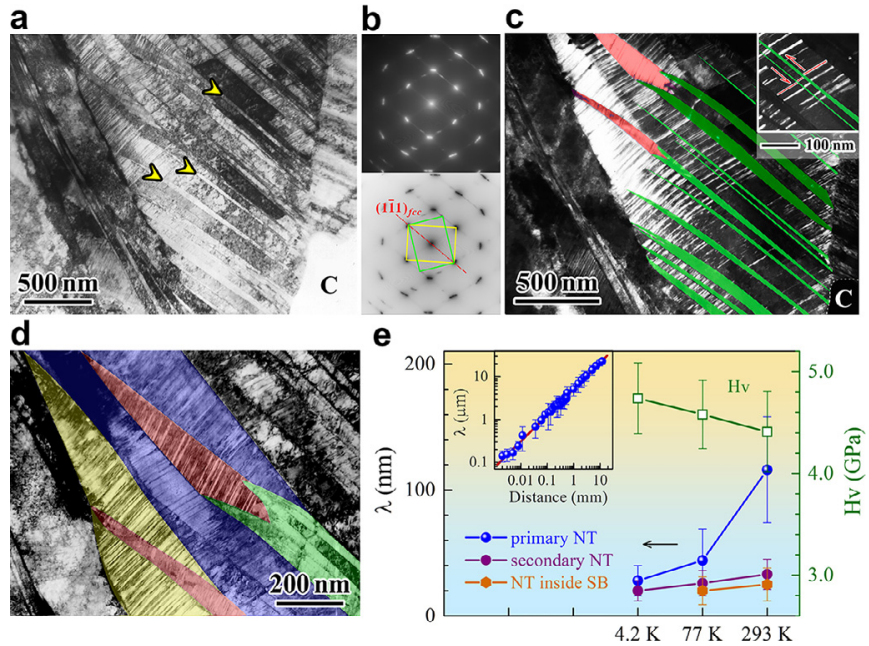

Fig. 4. (a) TEM micrograph showing lamellae of twin and matrix plates, together with thin SBs (indicated by arrows) in the grain ahead of crack tip (labeled by T) in a CVN sample after $A_{K}$ testing at RT. C: Crack tip. Yellow arrows: SBs. (b) Indexed selected-area electron diffraction pattern showing twin relationship of two sets. (c) Dark-field TEM micrograph showing secondary NTs of high density in front of the SBs. SBs are colored in green for easy recognition. Inset: shear-displacement of NTs by intersecting SBs. (d) Close-up view of (c) showing newly-induced SBs (crimson) at the tip of the original SB. Note NTs of high density inside SBs. Also note secondary NTs of high density in primary twin (light-blue)/matrix plate (yellow). (e) Statistic mean spacing $(\bar{\lambda})$ of primary NTs, and secondary NTs ahead of and inside the SBs, respectively, at $293 \mathrm{~K}, 77 \mathrm{~K}$, and $4.2 \mathrm{~K}$. Vickers micro-hardness (Hv): average value at $100 \mu \mathrm{m}$ away from the tip of the main propagating crack. (For interpretation of the references to colour in this figure legend, the reader is referred to the web version of this article.)

primary DT $[5,34,35]$, which also dominates at test temperatures below RT [2,5]. Strain hardening by DT was quantified using hardness mapping. An enhanced micro-hardness (Hv) (Fig. 3b and c) is seen across a large area of the vertical section of the CVN sample. The maximum $\mathrm{Hv}$ is as high as $5.2 \mathrm{GPa}$, locating ahead of the crack tip and along the crack path (fracture surface). A band of extremely high Hv (in crimson) indicates strong strain hardening. Further, NTs of high density are visible inside the band (Fig. 3d) much near the fracture surface (see inset). The profuse NTs dominate, as seen in Fig. 3e far away from the crack tip. Such a DT domination was known before at much lower deformation temperatures ( $77 \mathrm{~K}$ and $4.2 \mathrm{~K})[2,5]$.

The details as to how profuse DT dynamically interacts with an advancing SB are illustrated further in Fig. 4. Fig. 4a shows the lamella microstructure, consisting of both twin and matrix plates in a grain rather close to the tip of the main crack (labelled by C). Fig. $4 \mathrm{~b}$ is the selected area electron diffraction (SAED) pattern covering a few plates, and two sets of twin-matrix relationship are seen. These long twin plates are primary twins, emitting from GB and running across the whole grain. Of special note is the presence of a number of SBs of varying thickness (labelled by yellow arrows), which emit from the crack tip (marked by C) and already enter the grain interior. These SBs propagate almost parallel to the primary twins. This is the reason why diffraction spots of primary twin are stretched to a segment of arc (Fig. 4b). Fig. 4c is the darkfield TEM image showing the short secondary NTs inside either primary twins or matrix plates. It is visible that SBs, colored in green for easy recognition, has penetrated into either primary twin plates or matrix plates.

We emphasize here two concurrent effects of NTs on the propagation of SBs. Firstly, SBs end at the TBs of either short secondary NTs or long primary twin plates, see Fig. 4c. Inset shows the presence of shear displacements on both sides of NTs after the extending SBs cut through them [5,36]. All these indicate the blocking effect of TBs against the advancing SBs, similar to the effect on crack propagation $[9,10]$. The interior of the newly-generated SBs is observed to be full of NTs of high density, see Fig. 4d, with orientation obviously different from those outside. These NTs of high density, directly observed for the first time in the strain localized SB, would obviously strengthen and strain harden the SB. Taken together both effects, DT is clearly the dominant microstructural response underlying the deformation in and around a developing SB. This was not reported before to the best of our acknowledge.

Furthermore, the density of secondary NTs in an advancing SB is much higher at the front-end than that on the trailing end, see Fig. 4c. This indicates that these secondary NTs are in situ generated with a propagating SB. This is probably the result of the presence of plastic zone ahead of the tip of SB with intensified strain rates and stress concentration [37,38]. Besides, some de-twinning seems to have occurred after the SB passes by, as the twins on the trailing end are shorter with obviously lower density (Fig. 4c).

Fig. 4e shows the statistically average spacing $(\bar{\lambda})$ of NTs from TEM observations of at least several hundreds of NTs. All data are summarized, including primary and secondary NTs at three tested temperatures [5]. At RT, $\bar{\lambda}$ is $116 \mathrm{~nm}$ for primary NTs, $33 \mathrm{~nm}$ for secondary NTs in front of the SB, and $25 \mathrm{~nm}$ for secondary NTs inside the SB. The inset shows $\bar{\lambda}$ as a function of distance away from the crack tip. $\bar{\lambda}$ for secondary NTs is similar (20-40 nm) at three impact temperatures, all near the lower limit for previously reported nano-twinned metals [6-8]. Note that the profuse DT with nanometer $\bar{\lambda}$ should cause a high strain hardening rate. The highly increased Hv across a large plastic zone has been shown in Fig. $3 c$, which is a reason of the large energy consumption in Fig. 1c and $\mathrm{f}$. As expected, in the CrCoNi with a low SFE, the deformation twinning, and the high strain hardening associated with it, is advantageous for extending the plastic deformation zone and promoting fracture resistance. The DT is also what happens in and around the SB tip that suppresses it from turning into a runaway instability.

In summary, we report that the CrCoNi MEA exhibits high Charpy impact toughness across a wide temperature range. The root cause of this behavior is the large number of deformation nano-twins that are being embedded into the microstructure upon dynamic loading. DT carries massive plastic deformation and the accumulated NTs efficiently sustain high strain hardening, across a large plastic zone including in and around strain-localized deformation bands. As an emphasis, our detailed analysis reveals the presence of SBs under impact loading. We found that strain localization does happen during high strain rate deformation, and when SB emerges, two types of secondary NTs form around the developing SB. One type lies in front of each advancing SB (Fig. 4c). The SB has to cut through these NTs and often terminates there (Fig. 4c). In other words, these secondary NTs cause targeted blockage of SB propagation. The other type forms inside the interiors of SBs (Fig. 4d), strengthening and strain-hardening the SBs to reduce the tendency of micro-void nucleation in SBs and to delay the evolution from SB into a crack. This self-toughening alleviates softening in SBs that would otherwise lead to void formation and later crack propagation, allowing plastic strain to de-localize across the large plastic zone. Very recently, strain de-localization from SBs was also reported to occur in gradient nanostructure [39].

The profuse DT relieves locally high stresses and effectively consumes the imposed mechanical energy to cause large resistance to cracking, as reflected by the energy absorption behavior recorded in our instrumented Charpy test. We have shown above that DT is indeed also the agent that plays the harnessing role in holding off shear banding. Twin thickness/spacing down to nanometer scale is a critical factor: the smaller the $\bar{\lambda}$, the higher the work hardening rate [6], and hence the larger intrinsic toughening. To our knowledge, our report above is the first time that deformation twins (not pre-embedded growth twins) with 
thickness/spacing truly down to a few nanometers are directly observed to dominate for plastic deformation in bulk samples at RT. It is these DT events in and around the SBs that are highly effective in imposing control/constraints, when SBs begin to take shape occasionally in this MEA. This prevents any incipient SBs from turning into a runaway instability leading to catastrophic damage, even when the alloy is loaded under high strain rate impact conditions. If the SBs develop violently without control, there would be much less chance for spread-out deformation to consume energy throughout the plastic zone. Therefore, the suppression on SBs via deformation nanotwins, as illustrated in detail above, is a contributing factor to the overall toughness.

\section{Declaration of Competing Interest}

The authors declare that they have no known competing financial interests or personal relationships that could have appeared to influence the work reported in this paper.

\section{Acknowledgments}

X.W. is funded by the National Key R\&D Program of China (Grant nos. 2019YFA0200082 and 2017YFA0204402), the National Natural Science Foundation of China (Grant nos. 11572328, 11672313, and 11790293), and the Strategic Priority Research Program of the Chinese Academy of Sciences (Grant no. XDB22040503). E.M. acknowledges the support by US-NSF-DMR Grant no. 1804320.

\section{References}

[1] B. Gludovatz, A. Hohenwarter, D. Catoor, E.H. Chang, E.P. George, R.O. Ritchie, Science 345 (2014) 1153-1158.

[2] B. Gludovatz, A. Hohenwarter, K.V. Thurston, H. Bei, Z. Wu, E.P. George, R.O. Ritchie, Nat. Commun. 7 (2016) 10602.

[3] D. Li, Y. Zhang, Intermetallics 70 (2016) 24-28.

[4] S.Q. Xia, M.C. Gao, Y. Zhang, Mater. Chem. Phys. 210 (2018) 213-221.

[5] M.X. Yang, L.L. Zhou, C. Wang, P. Jiang, F.P. Yuan, E. Ma, X.L. Wu, Scr. Mater. 172 (2019) 66-71.
[6] L. Lu, X. Chen, X. Huang, K. Lu, Science 323 (2009) 607-610.

[7] K. Lu, L. Lu, S. Suresh, Science 324 (2009) 349-352.

[8] L. Lu, Z.S. You, K. Lu, Scr. Mater. 66 (2012) 837-842

[9] E.W. Qin, L. Lu, N.R. Tao, J. Tan, K. Lu, Acta Mater. 57 (2009) 6215-6225.

[10] L. Xiong, Z.S. You, L. Lu, Scr. Mater. 127 (2017) 173-177.

[11] A. Needleman, V. Tvergaard, J. Mech. Phys. Solids 35 (1987) 151-183.

[12] V. Tvergaard, Int. J. Solids Stru. 18 (1982) 659-672.

[13] K.K. Mathur, A. Needleman, V. Tvergaard Model, Simul. Mater. Sci. Eng. 2 (1994) 617.

[14] Z. Li, B. Wang, S. Zhao, R.Z. Valiev, K.S. Vecchio, M.A. Meyers, Acta Mater. 125 (2017) 210-218.

[15] J.F.C. Lins, H.R.Z. Sandim, H.J. Kestenbach, D. Raabe, K.S. Vecchio, Mater. Sci. Eng. A 457 (2007) 205-218.

16] Y.H. Zhang, Y. Zhuang, A. Hu, J.J. Kai, C.T. Liu, Scr. Mater. 130 (2017) 96-99.

[17] Z.Y. Liang, Y.Z. Li, M.X. Huang, Scr. Mater. 112 (2016) 28-31.

[18] X. Fu, X.L. Wu, Q. Yu, Mater. Today Nano 3 (2018) 48-53.

[19] M.X. Yang, D.S. Yan, F.P. Yuan, P. Jiang, E. Ma, X.L. Wu, Proc. Natl. Acad. Sci. U.S.A. 115 (2018) 7224-7229.

[20] D. Li, Y. Zhang, Intermetallics 70 (2016) 24-28.

[21] S.Q. Xia, M.C. Gao, Y. Zhang, Mater. Chem. Phys. 210 (2018) 213-221.

[22] R. Chaouadi, A. Fabry, in: D. Francois, A. Pineau (Eds.), From Charpy to Present Impact Testing, Elsevier, Oxford, 2002, pp. 103-117.

[23] A. Rossoll, C. Berdin, C. Prioul, Int. J. Fract. 115 (2002) 205-226.

[24] H. Kim, J. Park, J.E. Jung, S.S. Sohn, S. Lee, Mater. Sci. Eng. A 641 (2015) $340-347$.

[25] B. Hwang, T.H. Lee, S.J. Park, C.S. Oh, S.J. Kim, Mater. Sci. Eng. A 528 (2011) 7257-7266.

[26] S.V. Panin, P.O. Maruschak, I.V. Vlasov, B.B. Ovechkin, Theor. Appl. Frac. Mech. 83 (2016) 105-113.

[27] H. Kim, J. Park, M. Kang, S. Lee, Mater. Sci. Eng. A 649 (2016) 57-67.

[28] M. Divya, C.R. Das, S. Mahadevan, S.K. Albert, R. Pandian, S.K. Kar, A.K. Bhaduri, T. Jayakumar, Metall. Mater. Trans. A 46 (2015) 2554-2567.

[29] L. Lan, C. Qiu, D. Zhao, X. Gao, L. Du, Mater. Sci. Eng. A 529 (2011) 192-200.

[30] C.N. Li, G. Yuan, F.Q. Ji, D.S. Ren, G.D. Wang, Mater. Sci. Eng. A 665 (2016) 98-107.

[31] H. Tanino, M. Horita, K.I. Sugimoto, Metall. Mater. Trans. A 47 (2016) 2073-2080.

[32] B. Tanguy, J. Besson, R. Piques, A. Pineau, Eng. Fract. Mech. 72 (2005) 413-434

[33] S.H. Hashemi, Int. J. Pres. Vess. Pip. 85 (2008) 879-884.

[34] Y.T. Zhu, X.Z. Liao, X.L. Wu, Prog. Mater. Sci. 57 (2012) 1-62.

[35] X.L. Wu, Y.T. Zhu, Phys. Rev. Lett. 101 (2008) 025503.

[36] C.S. Hong, N.R. Tao, X. Huang, K. Lu, Acta Mater. 58 (2010) 3103-3116.

[37] M. Zhou, A.J. Rosakis, G. Ravichandran, J. Mech. Phys. Solids 44 (1996) 981-1006.

[38] Z. Zhang, R.J. Clifton, J. Mech. Phys. Solids 51 (2003) 1903-1922.

[39] F.P. Yuan, D.S. Yan, J.D. Sun, L.L. Zhou, Y.T. Zhu, X.L. Wu, Mater. Res. Lett. 7 (2019) 12-17. 